\title{
Non-Conjugated-Industrially-Produced-Trans Fatty in Lebanese Foods: The Case of Elaidic and Linolelaidic Acids
}

\author{
Maha Hoteit ${ }^{1}\left(\mathbb{D}\right.$, Edwina Zoghbi ${ }^{2}$, Alissar Rady ${ }^{2}$, Iman Shankiti ${ }^{2}$, Carla Ibrahim ${ }^{1,3}$ and Ayoub Al-Jawaldeh ${ }^{4, *}$ \\ 1 PHENOL Research Group (Public Health Nutrition Program-Lebanon), Faculty of Public Health, \\ Lebanese University, Beirut 6573, Lebanon; m.hoteit@ul.edu.lb (M.H.); carla.t.ibrahim@outlook.com (C.I.) \\ 2 Country Office for Lebanon, World Health Organization, Beirut 5391, Lebanon; zoghbi@who.int (E.Z.); \\ Radya@who.int (A.R.); shankitii@who.int (I.S.) \\ 3 Department of Nutrition and Food Sciences, Faculty of Arts and Sciences, Holy Spirit University of Kaslik, \\ Jounieh P.O. Box 446, Lebanon \\ 4 World Health Organization Regional Office for the Eastern Mediterranean, Cairo 11371, Egypt \\ * Correspondence: aljawaldeha@who.int
}

check for

updates

Citation: Hoteit, M.; Zoghbi, E.; Rady, A.; Shankiti, I.; Ibrahim, C.; Al-Jawaldeh, A. Non-ConjugatedIndustrially-Produced-Trans Fatty in Lebanese Foods: The Case of Elaidic and Linolelaidic Acids. Nutrients 2021, 13, 3664. https://doi.org/ $10.3390 /$ nu13103664

Academic Editors: Alessandra Durazzo, Massimo Lucarini and Laura den Hartigh

Received: 11 August 2021 Accepted: 13 October 2021 Published: 19 October 2021

Publisher's Note: MDPI stays neutral with regard to jurisdictional claims in published maps and institutional affiliations.

Copyright: (c) 2021 by the authors. Licensee MDPI, Basel, Switzerland. This article is an open access article distributed under the terms and conditions of the Creative Commons Attribution (CC BY) license (https:// creativecommons.org/licenses/by/ $4.0 /)$.

\begin{abstract}
To determine Industrially-Produced Trans fatty acids (IP-TFAs) distribution of Lebanese traditional foods, especially regarding Elaidic acid (EA; 9t18:1) and Linolelaidic acid (LEA; 9t12t18:2), a mapping exercise was enrolled between January 2019 and April 2021 in which 145 food samples of three categories (traditional dishes, Arabic sweets, and market food products) were analyzed using Gas chromatography methods. Results showed that about $93 \%$ of the products tested in Lebanon, between 2019 and 2021, met the World Health Organization recommendations, while about 7\% exceeded the limit. The mean level of the IP-TFAs Elaidic and Linolelaidic acid in most Traditional dishes $(0.9 \%)$, Arabic sweets $(0.6 \%)$, butter and margarine $(1.6 \%)$, and market foods $(0.52 \%)$ were relatively low compared with other countries. Despite that, the relative impact of IP-TFAs on heart diseases mortality in Lebanon is limited but unambiguously still substantial. The persistence of food products with high IP-TFAs levels threatens the health of Lebanese people. Fortunately, this problem is fairly easy to solve in Lebanon via proper legislation.
\end{abstract}

Keywords: industrially-produced trans fatty acids; Elaidic acid; Linolelaidic acid; traditional dishes; Arabic sweets; market foods; Lebanon

\section{Introduction}

The intake of Industrially-produced-Trans fatty acids (IP-TFAs) is associated with an increased risk of heart attacks and death from coronary heart disease (CHD) [1]. A 2\% absolute increase in energy intake from IP-TFAs has been associated with a $23 \%$ increase in cardiovascular risk [2]. In 2018, IP-TFAs elimination was identified as one of the priority targets in the World Health Organization (WHO) 13th General Programme of Work, which guides the five-year work of WHO in 2019-2023 [3]. Also, in 2018, the REPLACE action package was launched to help countries removing IP-TFAs from their food supplies [4]. In addition, WHO released additional resources in 2019 to support country actions, including six implementation modules and a live policy tracking map-the TFAs Country Score Card 1-to monitor global progress towards the 2023 target [3]. In 2020, WHO established an indicator that records whether countries have adopted WHO best-practice policies for eliminating IP-TFAs [5]. Around fifty-eight countries have introduced laws to date that will protect more than 3 billion people from TFAs by the end of 2021 [3]. However, more than 100 countries have yet to act to eliminate TFAs from their national food supply and make the world TFAs free by 2023 [3]. The European Region has the largest number of mandatory TFA limits in place and has had the most policy progress of all WHO regions since 2019. Since Denmark's effort (2004), Austria (2009), Iceland (2011), Hungary (2014), Norway (2014), Latvia (2018), Slovenia (2018) [6], and New Zealand (2008) have passed similar bestpractice regulations [7]. Switzerland, one of the first countries in Europe to take legal action 
to restrict TFA, has a TFA limit in oils and fats (2008) [6]. The Eastern Mediterranean Region (EMR), as well as Lebanon, have witnessed rapid modernization in the last thirty years that has led to a dramatic transformation affecting people's lifestyles and diets. The average intake of saturated fatty acids (SFAs) and IP-TFAs in EMR exceeded the WHO upper limits and was estimated to be $10.3 \%$ and $1.9 \%$ of total energy intake (EI), respectively [8]. The highest SFAs intake was reported in Djibouti, Kuwait, Saudi Arabia, Lebanon, and Yemen, while the highest intake of IP-TFAs was reported in Egypt and Pakistan [8]. According to recent national data, the proportion of coronary heart diseases (CHD) death due to IP-TFA intake is $9.4 \%$ ( $>0.5 \%$ energy) [5] and a high burden of NCDs, accounting for $91 \%$ of total annual deaths with CVDs responsible for $47 \%$ of total deaths [9] was observed in Lebanon. As a result, the urgent need for policy measures to protect cardiovascular health is more apparent than ever and presents a historic imperative to prioritize and invest in public health by adopting health-promoting policy measures, including industrially produced Trans fatty acids (IP-TFAs) elimination. Although limited data are available on IP-TFAs intake globally, a recent report estimated that the 2017 global market volume of partially hydrogenated vegetable oils (PHVO) - the main source of IP-TFAs in food-was approximately 13.6 million tones [10]. PHVO constitutes $25 \%$ to $45 \%$ of total fat [6]. Their removal from the global food supply could prevent up to 17 million deaths by 2040 and would be the first time an NCDs risk factor has been eliminated [11]. The most common non-conjugated IP-TFA in the human's daily diet are 18-carbon fatty acids with one double bond in the 9-carbon transposition or two double bonds in the 9 and 12 carbon, called Elaidic acid (EA; 9t18:1) and Linolelaidic acid (LEA; 9t12t18:2) respectively [12]. EA and LEA were associated with various health problems [13]. EA, which is the trans form of oleic acid (OA, C18:1 cis), is the principal IP-TFA found in PHVO and margarine. EA intake resulted in significant hyperlipidemia, inflammation, and fatty liver alterations [14]. LEA is an omega-6 TFA (9E,12E-9t12t18:2), principally discovered in foods with fried or high-heat cooking or PHVO [15]. It was suspected to enhance the adipogenic differentiation favoring obesity [15]. Moreover, LEA appeared to be potentially more detrimental than EA and LEA contributed to higher risks of sudden cardiac death compared with other TFAs [16]. Because IP-TFAs increases the risk of heart disease and are estimated to cause more than 500,000 deaths per year [3] and based on the WHO recommendation that IP-TFAs intake should not exceed $1 \%$ of total daily energy intake (equivalent to less than $2.2 \mathrm{~g} /$ day in a 2000-calorie diet), providing baseline information on dietary sources of IP-TFAs in Lebanon is a crucial stepstone to reduce the risk of death and hospitalization by CVDs and is one of the strategic interventions under the area of prevention and reduction of risk factors in the Regional Framework for Action on NCDs [17]. To our knowledge, this is the first national study that assesses the content of EA and LEA in food. The main objectives of this article are to:

Assess IP-TFAs levels, mainly EA and LEA in frequently consumed traditional dishes, Arabic sweets, processed foods, butter, and margarines in Lebanon.

Review of the findings retrieved from online databases on dietary sources of IP-TFAs in Lebanon and compare them with other countries.

Establish a steppingstone for required policies and regulations to mandate limits of IP-TFAs levels in foods imported or produced locally.

\section{Materials and Methods}

\subsection{Food Sampling}

A series of samples collections were conducted over the last two years. The 2019 samples collection, conducted in November 2019, was not centrally coordinated at the capital city Beirut but instead pooled data from five separate sources from the five main governorates in Lebanon (Beirut, Beqaa, Tripoli, Saida, and Mount Lebanon). In this sample collection, we collected thirty types of traditional dishes. Traditional composite dishes are defined as dishes consumed at main meals (i.e., lunch or dinner), containing ingredients from at least three of the five main food groups and requiring preparation 
using culinary skills [18-20]. A total of 30 traditional composite dishes were identified as most frequently consumed and hence were included for analysis. The names of the food dishes were reported in the current analysis considering the most familiar name used for the dish at a national level with respect to its ingredients. The ingredients of these traditional dishes were described in Hoteit et al. [18-20], and the food samples were collected from five different central kitchens in the 5 governorates listed above. The central kitchens were randomly chosen based on (1) their specialties in cooking homemade dishes, (2) their popularity in the area, (3) their implications in social entrepreneurship and women empowerment (e.g., household women who cook for these central kitchens). Consequently, the food samples were classified into 5 strata, per governorate area [20]. The samples were identified according to their frequency of consumption [21,22] and selected for IPTFAs analysis mainly for two non-conjugated fatty acids (EA and LEA). In contrast, the subsequent samples collections 2020, conducted in April, were centrally coordinated at Beirut having the broadest coverage in terms of products selected and had a sample of thirty-five types of Arabic sweets and forty-six types of market food products. The full methodology of food list identifications and food sampling is described elsewhere [18-20]. On the other hand, the 2021 sample collections, conducted in March, were nationally coordinated, with a coverage of 34 available types of butter and margarines purchased from all the Lebanese markets. Lot numbers were checked to ensure that each unit belonged to a different lot. The samples were stored, labeled, and analyzed before expiry dates. Samples were selected to include all types of butter and margarines in Lebanon. The analyses were carried out in duplicate for each sample. Thus, a composite sample from each type of food, according to each governorate, was prepared and analyzed. To further interpret current levels of IP-TFAs in Lebanese foods, product categories were compared with similar products found in other countries. A graphical scheme for the whole study is shown in Figure 1.
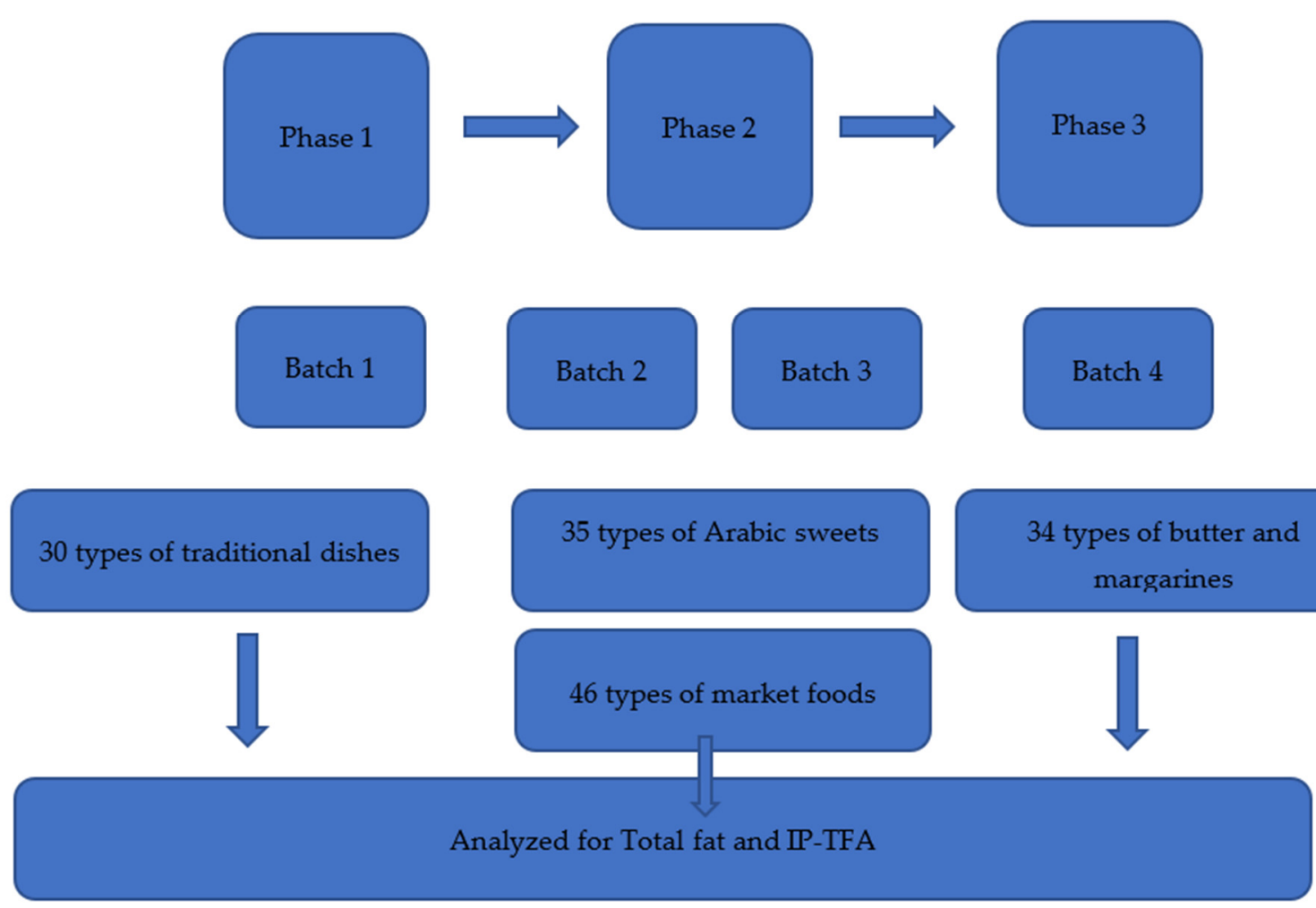

Figure 1. Graphical scheme of the current study. 


\subsection{Laboratory Analysis Protocol}

Around $500 \mathrm{~g}$ of each sample was mashed, then analyzed, and the remaining samples were kept frozen at $-18{ }^{\circ} \mathrm{C}$ for further analysis. The fatty acid profile was measured using gas chromatography. The IP-TFA analysis method was selected considering guidance from the technical committee at the Industrial Research Institute laboratories in Beirut and following standardized protocols. The Association of Official Analytical Chemists (AOAC) methods were used for the analysis of nutrients in food matrices [23].

Soxhlet extraction (Total fatty acids extraction):

The Roese-Gotlieb method was used in the investigation of the fat content [24]. Between 1-2 $\mathrm{g}$ of the dried food sample was filtered using a piece of filter paper. Later, it was wrapped and introduced into the soxhlet thimble. To avoid sample spilling, a cotton plug was placed at the top of the thimble. The soxhlet apparatus was assembled, and light petroleum, hexane, heptane, diethyl ether, or cyclohexane was used. The extraction was performed overnight. If solids from the thimble or sample were found in the solvent extract, a filtration before evaporation into another tarred flask or beaker was performed. Then it was dried to a constant weight, which was attained when successive $1 \mathrm{~h}$ drying periods showed additional loss of less than 0.05 fat. The percentage $(\%)$ fat was equal to $(\mathrm{g})$ fat $\times 100 \mathrm{~g}$ sample.

Fatty Acid Profile (saturated, unsaturated, trans):

The extracted fat of the sample that was obtained during fat determination was used to analyze the fatty acid profile. Between $200-500 \mathrm{mg}$ of the lipid sample was placed in a boiling flask with chips; then, $5 \mathrm{~mL}$ of $0.5 \mathrm{M}$ methanolic $\mathrm{KOH}$ was added. Esterification was performed by boiling under reflux for 3-5 min. An addition of $15 \mathrm{~mL}$ of esterification reagent $(2 \mathrm{~g} \mathrm{NaNH} 4+60 \mathrm{~mL}$ methanol $+3 \mathrm{~mL}$ conc sulfuric acid) through the condenser was performed, and then the sample was boiled for $15 \mathrm{~min}$. After cooling, there was an addition of $50 \mathrm{~mL}$ of distilled water and $25 \mathrm{~mL}$ of the solvent. The organic layer was isolated by means of a separatory funnel. Finally, the solvent layer was washed twice with distilled water.

Chromatographic analysis:

- Column: fatty acid methyl esters (FAME) length: 30 m, 0.32 ID

- Injection volume: $1 \mu \mathrm{L}$

- Injector temperature (PTV): injection: $60^{\circ} \mathrm{C}$ for $0.1 \mathrm{~min}$

- Transfer: ramp $10{ }^{\circ} \mathrm{C} / \mathrm{min}$ to $270{ }^{\circ} \mathrm{C}, 1 \mathrm{~min}$ hold

- Carrier flow (He): $2.0 \mathrm{~mL} / \mathrm{min}$

- Split flow: $20 \mathrm{~mL} / \mathrm{min}$ (split ratio: 20 )

- Detector temperature (flame ionization detection (FID) $280^{\circ} \mathrm{C}$

- Detector gases flows: Air 350 mL/min, Hydrogen 32 mL/min, Make-up (N2) 30 mL/min

Oven Program:

- Initial temperature: $100{ }^{\circ} \mathrm{C}$, hold $3 \mathrm{~min}$

- $\quad$ Ramp 1: $10^{\circ} \mathrm{C} / \mathrm{min}$ to $200^{\circ} \mathrm{C}$, hold $3 \mathrm{~min}$

- $\quad$ Ramp 2: $10^{\circ} \mathrm{C} / \min$ to $250{ }^{\circ} \mathrm{C}$, hold $5 \mathrm{~min}$

Using the chromatograph software Chromquest 4.2.34, USA, an integration of the areas under the peak was detected under the standards peaks. A calculation as percentage areas was done. The sum of trans fatty acids was calculated accordingly [24]. TFAs isomers were later on detected through SP-2560 $100 \mathrm{~m}$ capillary column $\left(180^{\circ} \mathrm{C}\right.$ isothermal, $\mathrm{H} 2$ at $1.0 \mathrm{~mL} / \mathrm{min}$ ) [23].

Statistical tests:

The study variables were presented as continuous variables and listed as reported values per 100g. Means and standard deviations were calculated for the group of traditional dishes, Arabic sweets, and other market products. T-test was used to compare the mean content of the group of foods in terms of EA and LEA. A $p$-value less than 0.05 indicates a statistically significant difference. Statistical analysis was conducted on IBM SPSS Statistics for Mac, Version 24, USA. 


\section{Results}

\subsection{Trans Fatty Acid Acids in Frequently Consumed Traditional Dishes}

The mean levels of total IP-TFAs (total of Elaidic acid and Linolelaidic acid) in the tested traditional dishes was equal to $0.9 \% \pm 0.62$ and ranged from less than 0.1 to $2.8 \mathrm{~g} / 100 \mathrm{~g}$ of total fat except for the dishes Riz a dajaj and Shawarma Lahma in which total IP-TFA exceeded $2 \%$ of the total fat (Table 1). The comparison between the mean values of the IP-TFA (EA and LEA) in the traditional dishes tested shows that EA was significantly higher than LEA in all traditional dishes $(p$-value $=0.00)$. Per each governorate, the mean level of total IP-TFAs in all dishes was $0.78 \pm 0.65 \%$ in Mount Lebanon (range: $<0.1-2.1 \%), 1.1 \% \pm 0.6$ in Beqaa (range: $<0.1-3 \%), 1 \pm 2.4 \%$ in Beirut (range: $<0.1-11.2 \%$ ), $1.2 \pm 0.9 \%$ in Tripoli (range: $<0.1-3.2 \%$ ) and $0.8 \pm 0.4 \%$ in Saida (range: $<0.1-2 \%$ ) (Data not shown).

Table 1. Total fat in $100 \mathrm{~g}$ of edible portions, total IP-TFAs and IP-TFAs (EA and LEA) in $100 \mathrm{~g}$ of fat of frequently consumed traditional dishes among all Lebanese governorates.

\begin{tabular}{|c|c|c|c|c|}
\hline \multirow[b]{2}{*}{ Dish } & \multirow[b]{2}{*}{ Total Fat $(\mathrm{g})$ in $100 \mathrm{~g}$} & \multirow[b]{2}{*}{$\begin{array}{c}\text { Total IP-TFA * per } 100 \mathrm{~g} \\
\text { of Total Fat }\end{array}$} & \multicolumn{2}{|c|}{ IP-TFAs in $100 \mathrm{~g}$ of Total Fat } \\
\hline & & & $\begin{array}{l}\text { Trans-C18:1n9t } \\
\text { (Elaidic Acid) }\end{array}$ & $\begin{array}{c}\text { Trans-C18:2n6t } \\
\text { (Linolelaidic Acid) }\end{array}$ \\
\hline Baba ghanouj & 9.44 & 0.74 & 0.66 & 0.08 \\
\hline Batata mahchi & 1.24 & 1.98 & 1.92 & 0.06 \\
\hline Borgul bil banadoura & 5.02 & 0.48 & 0.34 & 0.14 \\
\hline Chichbarak & 4.62 & 0.98 & 0.86 & 0.12 \\
\hline Falafel & 11.70 & 0.36 & 0.32 & 0.04 \\
\hline Fatayer sabanikh & 11.16 & 0.18 & 0.12 & 0.04 \\
\hline Fattat Hommos & 7.04 & 0.66 & 0.58 & 0.08 \\
\hline Fattoush & 2.94 & 0.8 & 0.5 & 0.3 \\
\hline Foul moudamas & 3.48 & 0.46 & 0.38 & 0.08 \\
\hline Hindbe bil zet & 10.70 & 0.18 & 0.18 & 0 \\
\hline Hommos bi tahini & 6.44 & 0.38 & 0.24 & 0.14 \\
\hline Kafta wa batata & 6.32 & 1.28 & 1.18 & 0.1 \\
\hline Kebba bil sayniya & 6.40 & 0.86 & 0.74 & 0.12 \\
\hline Koussa mahchi & 2.42 & 1.26 & 1.1 & 0.16 \\
\hline Lahm bil ajin & 8.96 & 0.34 & 0.22 & 0.12 \\
\hline Loubia bil zet & 5.68 & 0.52 & 0.46 & 0.06 \\
\hline Malfouf mahchi & 2.12 & 1.1 & 1.02 & 0.1 \\
\hline Moujadara & 5.80 & 0.36 & 0.36 & 0 \\
\hline Moghrabia & 3.94 & 0.86 & 0.76 & 0.1 \\
\hline Mousaka batinjan & 6.58 & 0.5 & 0.34 & 0.16 \\
\hline Riz a dajaj & 5.42 & 2.82 & 2.66 & 0.16 \\
\hline Riz bi lahma & 6.52 & 0.82 & 0.78 & 0.04 \\
\hline Sayadia & 6.48 & 0.22 & 0.18 & 0.04 \\
\hline Shawarma dajaj & 6.94 & 0.24 & 0.16 & 0.08 \\
\hline Shawarma lahma & 8.28 & 2.24 & 2.08 & 0.16 \\
\hline Tabboula & 4.24 & 0.38 & 0.26 & 0.12 \\
\hline Warak enab & 3.98 & 1.24 & 1.06 & 0.18 \\
\hline Yakhnat Bamia & 5.42 & 1.24 & 1.02 & 0.22 \\
\hline Yakhnat Fassoulia & 3.90 & 0.76 & 0.64 & 0.12 \\
\hline Yakhnat Mouloukhia & 4.28 & 1 & 0.8 & 0.2 \\
\hline
\end{tabular}

* This represents the sum of EA and LEA only. 


\subsection{Trans Fatty Acid Acids in Frequently Consumed Arabic Sweets}

The average of the total IP-TFAs in all samples of Arabic sweets was $0.6 \pm 0.3 \%$, predominantly from the EA type. (Table 2). Among 35 samples of Arabic sweets, none exceeded $2 \%$ as total IP-TFA in $100 \mathrm{~g}$ of total fat. The comparison between the mean values of the IP-TFA (EA and LEA) in the Arabic sweets tested shows that EA was significantly higher than LEA in Arabic sweets ( $p$-value $=0.00$ ).

Table 2. Total fat in $100 \mathrm{~g}$ of edible portions, total IP-TFAs and IP-TFAs (EA and LEA) in $100 \mathrm{~g}$ of total fat of frequently consumed Arabic sweets.

\begin{tabular}{|c|c|c|c|c|}
\hline \multirow[b]{2}{*}{ Name } & \multirow[b]{2}{*}{ Total Fat $(\mathrm{g})$ in $100 \mathrm{~g}$} & \multirow[b]{2}{*}{$\begin{array}{c}\text { Total IP-TFAs per } 100 \mathrm{~g} \\
\text { of Total Fat * }\end{array}$} & \multicolumn{2}{|c|}{ IP-TFAs in $100 \mathrm{~g}$ of Total Fat } \\
\hline & & & $\begin{array}{l}\text { Trans-C18:1n9t } \\
\text { (Elaidic Acid) }\end{array}$ & $\begin{array}{c}\text { Trans-C18:2n6t } \\
\text { (Linolelaidic Acid) }\end{array}$ \\
\hline Baklava Mixed & 23.45 & 0.25 & 0.2 & 0.05 \\
\hline Baklava Mixed Light & 20.5 & 0.3 & 0.3 & 0 \\
\hline Halawat El Jiben & 8.95 & 1.2 & 1.05 & 0.15 \\
\hline katayef Kashta & 6.65 & 0.9 & 0.65 & 0.25 \\
\hline Kounafa bil jiben & 12.25 & 0.4 & 0.25 & 0.15 \\
\hline Maakaroun & 12 & 0.1 & 0.1 & 0 \\
\hline Maamoul Tamer & 17.4 & 0.4 & 0.25 & 0.15 \\
\hline Maamoul mad Kashta & 10.65 & 0.4 & 0.25 & 0.15 \\
\hline Maamoul mad joz & 19.2 & 0.45 & 0.4 & 0.05 \\
\hline Maamoul joz & 21.5 & 0.85 & 0.75 & 0.1 \\
\hline Mafrouka Kashta & 13.25 & 0.4 & 0.2 & 0.2 \\
\hline Mafroukeh fostok & 10.6 & 0.6 & 0.5 & 0.1 \\
\hline Moushabak & 20.1 & 0.4 & 0.4 & 0 \\
\hline Nammoura & 5.9 & 1.5 & 1.3 & 0.2 \\
\hline Osmaliya & 16.25 & 0.5 & 0.4 & 0.1 \\
\hline Saniora & 23.8 & 1.15 & 0.85 & 0.3 \\
\hline Sfouf & 12.45 & 1.45 & 1.2 & 0.25 \\
\hline Barazik & 16.5 & 0.5 & 0.5 & 0 \\
\hline Boundoukia & 19.5 & 0.3 & 0.3 & 0 \\
\hline Daoukia & 14.8 & 0.4 & 0.3 & 0.1 \\
\hline Foustoukia & 20.4 & 0.4 & 0.4 & 0 \\
\hline Ghourayba & 0.325 .8 & 0.6 & 0.4 & 0.2 \\
\hline Ish el bulbul & 25.1 & 0.2 & 0.2 & 0 \\
\hline kallaj kashta & 9.6 & $<0.1$ & - & - \\
\hline Karabij joz maa crema & 18.8 & 0.4 & 0.2 & 0.2 \\
\hline kounafa kashta maa kaak & 10 & 0.4 & 0.1 & 0.3 \\
\hline Maakroun wa Moushabak & 13.7 & $<0.1$ & - & - \\
\hline Maamoul fostok & 19.1 & 0.7 & 0.5 & 0.2 \\
\hline Madlouka & 11.5 & 0.6 & 0.5 & 0.1 \\
\hline Moufattaka & 20.7 & $<0.1$ & - & - \\
\hline Mouhallabiya & 4 & 0.5 & 0.1 & 0.4 \\
\hline Riz bil Halib & 4.4 & $<0.1$ & - & - \\
\hline Shaaybiyat & 16.1 & $<0.1$ & - & - \\
\hline Ward el sham & 14.2 & 0.5 & 0.5 & 0 \\
\hline Znoud El sitt & 12.3 & $<0.1$ & - & - \\
\hline
\end{tabular}




\subsection{Trans Fatty Acid in Market Foods}

3.3.1. Cereals and Breads Group

In the group of cereals and breads, the mean level of total IP-TFAs was less than $2 \%$ of total fat except for pain au lait (total IP-TFAs: $3.8 \%$ ), which is usually prepared from wheat, milk, and butter or ghee to be consumed frequently by children as a sandwich (Tables 3 and 4 ).

Table 3. Total fat in $100 \mathrm{~g}$ of edible portions, total IP-TFAs and IP-TFAS (EA and LEA) in $100 \mathrm{~g}$ of total fat of Market food products collected from Lebanese markets.

\begin{tabular}{|c|c|c|c|c|}
\hline \multirow[b]{2}{*}{ Product } & \multirow[b]{2}{*}{ Total Fat $(\mathrm{g})$ in $100 \mathrm{~g}$} & \multirow[b]{2}{*}{$\begin{array}{c}\text { Total IP-TFA per } 100 \mathrm{~g} \\
\text { of Total Fat }\end{array}$} & \multicolumn{2}{|c|}{ IP-TFAs in $100 \mathrm{~g}$ of Total Fat } \\
\hline & & & $\begin{array}{l}\text { Trans-C18:1n9t } \\
\text { (Elaidic Acid) }\end{array}$ & $\begin{array}{c}\text { Trans-C18:2n6t } \\
\text { (Linolelaidic Acid) }\end{array}$ \\
\hline Arabic Bread-White & 2.3 & $<0.1$ & - & - \\
\hline Arabic Bread-Whole wheat & 4 & $<0.1$ & - & - \\
\hline Baguette & 0.5 & $<0.1$ & - & - \\
\hline Biscuits Chocolate Quinoa & 13.4 & 0.1 & 0.1 & - \\
\hline Biscuits Digestive & 17.1 & 0.3 & 0.1 & 0.2 \\
\hline Biscuits Digestive Light & 13.8 & 0.3 & 0.1 & 0.2 \\
\hline Biscuits with cream & 15.5 & $<0.1$ & - & - \\
\hline Breakfast Cereals & 2.1 & $<0.1$ & - & - \\
\hline $\begin{array}{l}\text { Breakfast } \\
\text { Cereals-Chocolate }\end{array}$ & 2.4 & 0.3 & 0.1 & 0.2 \\
\hline Butter ( $n=17$ samples) & 80 & 0.8 & 0.6 & 0.2 \\
\hline Cake with Cream & 16.1 & $<0.1$ & - & - \\
\hline Chocolate Dark & 33.6 & $<0.1$ & - & - \\
\hline Chocolate Milk-1 & 36.6 & 0.1 & 0.1 & - \\
\hline Chocolate Milk-2 & 35 & $<0.1$ & - & - \\
\hline Coffee without cardamon & 16.8 & 0.2 & 0.1 & 0.1 \\
\hline Coffee with cardamon & 17.7 & 0.3 & 0.1 & 0.2 \\
\hline Corn Oil & 100 & $<0.1$ & - & - \\
\hline Croissant Zaatar-1 (cheap) & 16.1 & 0.7 & 0.4 & 0.3 \\
\hline $\begin{array}{l}\text { Croissant zaatar-2 } \\
\text { (expensive) }\end{array}$ & 22.5 & 0.1 & 0.1 & \\
\hline De-hulled Pumpkin Seeds & 50.6 & 0.6 & 0.3 & 0.3 \\
\hline De-hulled Sunflower Seeds & 52.5 & 0.7 & 0.3 & 0.4 \\
\hline Doughnuts & 19.6 & 0.5 & 0.5 & - \\
\hline English Cake-Chocolate & 18.6 & 2.6 & 2.6 & - \\
\hline Margarines $(n=18)$ & 100 & 2.4 & 2.2 & 0.2 \\
\hline Halawa & 25.5 & 0.4 & 0.4 & - \\
\hline Halawa Light & 29.9 & 1.3 & 1.1 & 0.2 \\
\hline Hot Chocolate Powder & 5.4 & 0.3 & 0.3 & - \\
\hline Instant Coffee & 10.8 & 0.2 & 0.2 & - \\
\hline Kaak asrouni ** & 1.5 & $\operatorname{Tr}$ & - & - \\
\hline Kaak debes and Cacao ${ }^{* * *}$ & 11.9 & 0.3 & 0.2 & 0.1 \\
\hline Kaak korshalli **** & 6.9 & 0.5 & 0.5 & - \\
\hline
\end{tabular}


Table 3. Cont.

\begin{tabular}{|c|c|c|c|c|}
\hline \multirow[b]{2}{*}{ Product } & \multirow[b]{2}{*}{ Total Fat $(\mathrm{g})$ in $100 \mathrm{~g}$} & \multirow[b]{2}{*}{$\begin{array}{c}\text { Total IP-TFA per } 100 \mathrm{~g} \\
\text { of Total Fat }\end{array}$} & \multicolumn{2}{|c|}{ IP-TFAs in $100 \mathrm{~g}$ of Total Fat } \\
\hline & & & $\begin{array}{l}\text { Trans-C18:1n9t } \\
\text { (Elaidic Acid) }\end{array}$ & $\begin{array}{c}\text { Trans-C18:2n6t } \\
\text { (Linolelaidic Acid) }\end{array}$ \\
\hline Mixed Kernels & 53.6 & $<0.1$ & & - \\
\hline Mixed Nuts & 25.7 & 0.3 & 0.2 & 0.1 \\
\hline Olive Oil & 100 & $<0.1$ & - & - \\
\hline Pain au Lait & 3.8 & 2.7 & 2.7 & - \\
\hline Petit Fours-1 (cheap) & 25.6 & 0.2 & 0.2 & - \\
\hline Petit Fours-2 (expensive) & 29.6 & 0.2 & - & 0.2 \\
\hline Potato Chips-1 & 29.9 & 0.1 & 0.1 & - \\
\hline Potato Chips-2 & 15.4 & 0.3 & 0.2 & 0.1 \\
\hline Potato Chips Light-1 & 26.9 & 0.1 & 0.1 & - \\
\hline Potato Chips light-2 & 22.9 & 0.3 & 0.3 & - \\
\hline Sunflower Oil & 100 & $<0.1$ & - & - \\
\hline Tahina & 59.4 & 0.1 & - & 0.1 \\
\hline Tuna Packed in Oil & 6.8 & 0.3 & 0.1 & 0.2 \\
\hline Tuna Packed in Water & 0.5 & 0.6 & 0.6 & - \\
\hline Wafer-Chocolate-1 & 21.7 & $<0.1$ & - & - \\
\hline $\begin{array}{l}\text { Wafer-Chocolate-2 } \\
\text { (manufactured in Lebanon) }\end{array}$ & 24.2 & 6.5 & 6.2 & 0.3 \\
\hline
\end{tabular}

** Kaak Asrouni: type of Lebanese street bread. ${ }^{* * *}$ Kaak Debes and Cacao: Cacao cookies with molasses. ${ }^{* * *}$ Kaak korshalli: toast bagel (elongated shape).

Table 4. Industrially-Produced trans fatty acids (EA and LEA) in $100 \mathrm{~g}$ of total fat per food groups among different countries.

\section{IP-TFAs}

\section{Countries}

France

New Zealand Margarines and table spreads: 12.3

Margarine/butter blends: 8.3

Butters: 5.2

\begin{tabular}{lc}
\hline Spain & Spanish margarines: 8.17 \\
\hline \multirow{2}{*}{ Bulgaria } & Imported margarines: 8.4 \\
& Bulgarian margarines: 1.12 \\
\hline
\end{tabular}

$\begin{array}{cc}\text { Turkey } & \text { Margarine tub: } 3.85 \\ \text { Margarine stick: } 16.88\end{array}$

Breakfast cereal: 6.75

Korea

Trans-C18:1n9t
(Elaidic Acid) (\%)

Cake: 24.43

Roasted bread: 33.1

ted bread: 25.8

30.3

Cookies 38.9
Cereals: 28.9
Trans-C18:2n6t

(Linolelaidic Acid) (\%)
Cream-filled biscuit: 15.57

Cream-stuffed cake: 20.96

Canned coffee: 2.3
Margarines and table spreads (low trans): 0.1

Margarines and table spreads: 1.3

Margarine/butter blends: 1.6

Butters: 1.7

Spanish margarines: 0.49

Margarine tub: 0

Margarine stick: 2.09

Breakfast cereal: 0.25

Cream-filled biscuit: 0.43

Cream-stuffed cake: 0.66

Canned coffee: 0.3 
Table 4. Cont.

\begin{tabular}{|c|c|c|}
\hline \multicolumn{3}{|c|}{ IP-TFAs } \\
\hline Countries & $\begin{array}{l}\text { Trans-C18:1n9t } \\
\text { (Elaidic Acid) (\%) }\end{array}$ & $\begin{array}{c}\text { Trans-C18:2n6t } \\
\text { (Linolelaidic Acid) (\%) }\end{array}$ \\
\hline New Zealand & $\begin{array}{c}\text { Biscuits and cakes: } 0.9 \\
\text { Margarines/spreads: } 4.9 \\
\text { Chocolate: } 1.1 \\
\text { Snack bars: } 0.4 \\
\text { Pies and pastry: } 3.7 \\
\text { Partially cooked chips/wedges: } 2.5\end{array}$ & $\begin{array}{c}\text { Biscuits and cakes: } 0 \\
\text { Margarines/spreads: } 0.1 \\
\text { Chocolate: } 0 \\
\text { Snack bars: } 0.1 \\
\text { Pies and pastry: } 0.4 \\
\text { Partially cooked chips/wedges: } 0.4\end{array}$ \\
\hline Pakistan & $\begin{array}{l}\text { Margarines: } 7.89 \\
\text { Butter: } 3.82\end{array}$ & Margarines: 0.45 \\
\hline Turkey & Margarines and shortenings: 10.55 & - \\
\hline Canada & $\begin{array}{l}\text { Tub margarines: } 3.4 \\
\text { Print margarines: } 5.5\end{array}$ & $\begin{array}{c}\text { Tub margarines: } 0.1 \\
\text { Print margarines: } 0.3\end{array}$ \\
\hline Costa Rica & $\begin{array}{c}\text { Corn oil: } 0.35 \\
\text { Sunflower oil: } 0.28 \\
\text { Olive oil: } 0.26 \\
\text { Margarines: } 10.15 \\
\text { Butter: } 5.1 \\
\text { Mixed nuts: } 0.2 \\
\text { Canned tuna (oil): } 0.54 \\
\text { Canned tuna (water): } 1.07 \\
\text { Nondairy coffee creamer: } 30.84\end{array}$ & $\begin{array}{c}\text { Corn oil: } 0.07 \\
\text { Sunflower oil: } 0.09 \\
\text { Olive oil: } 0 \\
\text { Margarines: } 0.35 \\
\text { Butter: } 0.23 \\
\text { Mixed nuts: } 0 \\
\text { Mayonnaise: } 0.02 \\
\text { Canned tuna (oil): } 0.08 \\
\text { Canned tuna (water): } 0 \\
\text { Nondairy coffee creamer: } 1.15\end{array}$ \\
\hline Korea & $\begin{array}{c}\text { Breakfast cereal: } 0.5 \\
\text { Cream-filled biscuit: } 2.4 \\
\text { Cream-stuffed cake: } 1.36 \\
\text { Canned coffee: } 2.3\end{array}$ & $\begin{array}{c}\text { Breakfast cereal: } 0.3 \\
\text { Cream-filled biscuit: } 0.25 \\
\text { Cream-stuffed cake: } 0.26 \\
\text { Canned coffee: } 0.7\end{array}$ \\
\hline Pakistan & Margarines: 19.48 & Margarines: 0.49 \\
\hline Brazil & $\begin{array}{l}\text { Regular dark Chocolate: } 0.078 \\
\text { Regular chocolate: } 0.075\end{array}$ & - \\
\hline Germany & $\begin{array}{c}\text { Margarines/spreads: } 0.2 \\
\text { Shortenings/cooking fats: } 0.51 \\
\text { Doughnuts: } 2.07 \\
\text { Chocolate products: } 0.44 \\
\text { Biscuits: } 0.18 \\
\text { Instant coffee products: } 0.36 \\
\text { Butter: } 0.23\end{array}$ & - \\
\hline Mexico & $\begin{array}{l}\text { Spreadable margarines: } 4.73 \\
\text { Stick margarines: } 7.4\end{array}$ & $\begin{array}{l}\text { Spreadable margarines: } 0.39 \\
\text { Stick margarines: } 0.94\end{array}$ \\
\hline Turkey & $\begin{array}{l}\text { Potato crisps: } 0.13 \\
\text { Corn crisps: } 0.24 \\
\text { Cocoa cakes: } 0.37 \\
\text { Chocolate cakes: } 0.55 \\
\text { Cream cakes: } 0.78 \\
\text { Fruity cakes: } 1\end{array}$ & $\begin{array}{c}\text { Potato crisps: } 0.15 \\
\text { Corn crisps: } 0.16 \\
\text { Cocoa cakes: } 0.11 \\
\text { Mosaic cakes: } 0.05 \\
\text { Chocolate cakes: } 0.08 \\
\text { Cream cakes: } 0.24 \\
\text { Hazelnut-cocoa cakes: } 0.14 \\
\text { Fruity cakes: } 0.09\end{array}$ \\
\hline India & $\begin{array}{c}\text { Biscuit: } 0.01 \\
\text { Pastry: } 0.85 \\
\text { Cake: } 1.92 \\
\text { Bread: } 0.18 \\
\text { Bun: } 1.31\end{array}$ & $\begin{array}{c}\text { Biscuit: } 0 \\
\text { Pastry: } 0 \\
\text { Cake: } 0.04 \\
\text { Bread: } 0.007 \\
\text { Bun: } 0.03\end{array}$ \\
\hline
\end{tabular}


Table 4. Cont.

\begin{tabular}{|c|c|c|}
\hline \multirow[b]{2}{*}{ Countries } & \multicolumn{2}{|c|}{ IP-TFAs } \\
\hline & $\begin{array}{l}\text { Trans-C18:1n9t } \\
\text { (Elaidic Acid) (\%) }\end{array}$ & $\begin{array}{c}\text { Trans-C18:2n6t } \\
\text { (Linolelaidic Acid) (\%) }\end{array}$ \\
\hline Iran & $\begin{array}{c}\text { Cakes: } 18 \\
\text { Cream biscuits: } 12 \\
\text { Simple biscuits: } 9 \\
\text { Simple chocolates: } 5 \\
\text { Potato chips: } 10 \\
\text { Margarine: } 3.2\end{array}$ & $\begin{array}{c}\text { Cakes: } 0 \\
\text { Cream biscuits: } 2 \\
\text { Simple biscuits: } 2 \\
\text { Simple chocolates: } 0 \\
\text { Potato chips: } 4 \\
\text { Margarine: } 0.9\end{array}$ \\
\hline UK & $\begin{array}{c}\text { Breakfast cereal products: } 0.03 \\
\text { Margarine, hard block: } 0.05 \\
\text { Potato chips, takeaway: } 0.97 \\
\text { Potato chips, fine cut, takeaway: } 0.08 \\
\text { Potato chips, oven baked: }<0.02 \\
\text { Potato snacks and corn snacks: } 0.08 \\
\text { Confectionery, non-chocolate: } 0.05 \\
\text { Confectionery, chocolate: } 0.08 \\
\text { Butter, spreadable: } 0.22\end{array}$ & - \\
\hline Malaysia & $\begin{array}{c}\text { Cakes: }<0.001 \\
\text { Doughnuts: }<0.001 \\
\text { Croissants: }<0.001-0.02 \\
\text { White bread: }<0.001 \\
\text { Whole grain bread: }<0.001 \\
\text { Buns: }<0.001 \\
\text { Cream crackers: }<0.001-0.33 \\
\text { Chocolate biscuits: }<0.001 \\
\text { Potato chips: }<0.001-0.87 \\
\text { Chocolate bars: }<0.001 \\
\text { Chocolate wafers: }<0.001-0.38 \\
\text { Olive oil: } 0.79 \\
\text { Blended oil (canola, soybean and olive): } 0.82 \\
\text { Soybean oil: } 1.76 \\
\text { Palm oil: } 1.79 \\
\text { Corn oil: }<0.001 \\
\text { Coco-coated cereal: } 1.57 \\
\text { Corn cereal: }<0.001 \\
\text { Cereal beverages: }<0.001\end{array}$ & $\begin{array}{c}\text { Cakes: }<0.001 \\
\text { Doughnuts: }<0.001 \\
\text { Croissants: }<0.001 \\
\text { White bread: } 3.12 \\
\text { Whole grain bread: }<0.001 \\
\text { Buns: }<0.001-1.21 \\
\text { Cream crackers: }<0.001 \\
\text { Chocolate biscuits: }<0.001-0.02 \\
\text { Potato chips: }<0.001-1.02 \\
\text { Chocolate bars: }<0.001-0.54 \\
\text { Chocolate wafers: }<0.001 \\
\text { Olive oil: }<0.001 \\
\text { Blended oil (canola, soybean and olive): } 3.24 \\
\text { Soybean oil: } 4.06 \\
\text { Palm oil: }<0.001 \\
\text { Corn oil: } 2.13 \\
\text { Coco-coated cereal: }<0.001 \\
\text { Corn cereal: } 4.82 \\
\text { Cereal beverages: }<0.001-6.60\end{array}$ \\
\hline Iran & $\begin{array}{l}\text { Liquid frying oils: } 0.08 \\
\text { Solid frying oils: } 1.26\end{array}$ & $\begin{array}{l}\text { Liquid frying oils: } 0.01 \\
\text { Solid frying oils: } 0.03\end{array}$ \\
\hline Saudi Arabia & Margarines and shortenings: 5.43 & Margarines and shortenings: 1.49 \\
\hline Iran & Margarines: 5.99 & Margarines: 0.66 \\
\hline Iran & $\begin{array}{l}\text { Biscuit: } 12.86 \\
\text { Cake: } 6.95 \\
\text { Shortcake: } 3.38 \\
\text { Donuts: } 3.29 \\
\text { Bread tan: } 2.99 \\
\text { Baklava: } 2.5 \\
\text { Chocolate: } 1.24 \\
\text { Chips: } 0.61 \\
\text { Snack: } 0.52\end{array}$ & - \\
\hline Iran & $\begin{array}{l}\text { Edible oils: } 0.07 \\
\text { Margarines: } 5.3\end{array}$ & - \\
\hline India & Cakes: 3.93 & Cakes: 2.82 \\
\hline
\end{tabular}


Table 4. Cont.

\begin{tabular}{|c|c|c|}
\hline & \multicolumn{2}{|c|}{ IP-TFAs } \\
\hline Countries & $\begin{array}{l}\text { Trans-C18:1n9t } \\
\text { (Elaidic Acid) (\%) }\end{array}$ & $\begin{array}{c}\text { Trans-C18:2n6t } \\
\text { (Linolelaidic Acid) (\%) }\end{array}$ \\
\hline Lebanon & $\begin{array}{l}\text { Cakes: } 1.7 \\
\text { Biscuits: } 3.7 \\
\text { Croissant: } 2.7 \\
\text { Wafers: } 5.6\end{array}$ & $\begin{array}{l}\text { Cakes: } 0.1 \\
\text { Biscuits: } 0.1 \\
\text { Croissant: } 0.1 \\
\text { Wafers: } 0.1\end{array}$ \\
\hline Slovenia & Margarines and shortenings: 34.63 & Margarines and shortenings: 21.38 \\
\hline Serbia & $\begin{array}{l}\text { Crackers: } 0.9 \\
\text { Chips and flips: } 5.34 \\
\text { Fried corn nuts: } 1.7\end{array}$ & $\begin{array}{c}\text { Crackers: } 0.5 \\
\text { Chips and flips: } 0.152 \\
\text { Fried corn nuts: } 0.1\end{array}$ \\
\hline Poland & $\begin{array}{l}\text { Biscuits: } 2.81 \\
\text { French pastry cookies: } 1.65\end{array}$ & $\begin{array}{c}\text { Biscuits: } 0.21 \\
\text { French pastry cookies: } 0.275\end{array}$ \\
\hline Tunisia & $\begin{array}{l}\text { Margarines: } 4.47 \\
\text { Frying oil: } 0.14\end{array}$ & $\begin{array}{l}\text { Margarines: } 4.47 \\
\text { Frying oil: } 0.24\end{array}$ \\
\hline $\begin{array}{l}\text { Lebanon } 2021 \text { (current } \\
\text { study) }\end{array}$ & $\begin{array}{l}\text { Traditional dishes: } 0.7 \\
\text { Arabic sweets: } 0.5 \\
\text { Butter and margarines: } 1.4 \\
\text { Biscuits, doughnuts, cake: } 0.4 \\
\text { Cereals and breads group: } 0.3 \\
\text { Tuna: } 0.35 \\
\text { Chocolate and chocolate wafers: } 1.26 \\
\text { Cooking oils: } 0 \\
\text { Coffee and instant coffee: } 0.2 \\
\text { Chips, nuts and seeds: } 0.2 \\
\text { Tahina and Halawa: } 0.5\end{array}$ & $\begin{array}{l}\text { Traditional dishes: } 0.9 \\
\text { Arabic sweets: } 0.6 \\
\text { Butter and margarines: } 1.6 \\
\text { Biscuits, doughnuts, cake: } 0.5 \\
\text { Cereals and breads group: } 0.3 \\
\text { Tuna: } 0.45 \\
\text { Chocolate and chocolate wafers: } 1.3 \\
\text { Cooking oils: } 0 \\
\text { Coffee and instant coffee: } 0.25 \\
\text { Chips, nuts and seeds: } 0.3 \\
\text { Tahina and Halawa: } 0.6\end{array}$ \\
\hline
\end{tabular}

\subsubsection{Butter and Margarines}

Particular attention was given to the margarine group as it is used as an ingredient and therefore amongst the main sources of IP-TFAs in processed foods. The average of total IP-TFAs in 18 margarines used frequently in Lebanon was $2.4 \pm 0.4 \%$ (Table 3 ) with a range between $<0.1 \%$ and $11.8 \%$ (Data not shown). The dominant IP-TFA was EA in almost all these products (Table 3). Within the group of butter, none of the samples exceeded $2 \%$ of total fat. The average of total IP-TFAs in the butter and margarines group was $1.6 \pm 0.6 \%$ of total fat in which EA predominates in these products. Generally, the level of total IP-TFAs in cooking oils, Halawa and Tahina was negligible (Tables 3 and 4).

\subsubsection{Snacks and Processed Foods}

As for the group of biscuits, doughnuts, and cakes group, negligible amounts of IP-TFAs were found in these products (Average: $0.5 \% \pm 0.2$ ) (Table 4). On the other hand, the unlabeled English cake (chocolate flavor) had an apparently high amount of total IP-TFAs (2.6\% in the total fat) in which EA was dominantly available (Table 3). Despite being unable to discuss the fat type used in unlabeled samples, based on this data, partially hydrogenated fats were certainly present in high amounts.

The data on chocolate products presented an amount of $1.3 \% \pm 0.3$ as total IP-TFAs (Figure 1), except for the case of wafer-coated chocolate originally manufactured in Lebanon which contains a level of $6.5 \%$ (Table 3).

According to Tables 3 and 4, it appears that all samples of potato chips, nuts, seeds, coffee, instant coffee, and packed tuna contained low amounts of total IP-TFAs that are below $2 \%$ of total fat. When comparing the mean values of the IP-TFA (EA and LEA) in the market foods, EA and LEA didn't show any significant difference ( $p$-value $=0.16$ ). 


\section{Discussion}

Industrially-Produced Trans fatty acid content in frequently consumed foods in Lebanon compared with different countries.

The available data, the first of its kind in Lebanon, demonstrate that categories with the highest IP-TFAs levels included Riz a dajaj, Shawarma Lahma, Pain au lait, English cake, Chocolate wafers, and margarines. About $93 \%$ of the products tested in Lebanon, between 2019 and 2021, met the WHO recommendations (less than $2 \%$ of Trans fatty acid in total fat), while about $7 \%$ exceeded the limit. As per Tables 1-3, all in all, EA was dominant in almost all the analyzed samples and its higher amount indicates that hydrogenated oils were a major contributor in the processing of food products or baking and cooking meals. In comparison to other countries all over the globe, a broad range of EA was observed in many food products (Table 4). For instance, the mean level of EA in Baklava (0.2\%) was relatively low in our study in comparison with the content of EA in Baklava in Iran (2.5\%) [25]. Furthermore, our findings showed that the mean levels of EA in cakes (2.6\%) was much lower than the content of EA found in cakes in France (18.5-25.6\%) [26], Iran (6.95-18\%) [25-27], Poland (7.95\%) [28], India (1.92-3.93\%) [29], and higher than EA cake content tested in Lebanon in 2015 (1.7\%) [30], Korea (1.36\%) [31], Turkey (0.37-1.43\%) [32], New Zealand (0.9\%) [7], and Malaysia $(<0.001 \%)$ [33] (Table 4). In addition, the mean levels of EA in biscuits in Iran (9-12.86\%) [27], Lebanon 2015 (3.7\%) [30], Poland (2.81\%) [28], Korea (2.4\%) [31], New Zealand (0.9\%) [7], and Germany (0.18\%) [34] were higher than our results $(0.1 \%)$, except for Malaysia $(<0.001 \%)$ [33] and India $(0.01 \%)$ [29] (Table 4). As for the breakfast cereals, the mean level of EA in our study $(0.1 \%)$ was much lower than in France (28.9-32.4\%) [26] and Korea (0.5-6.75\%) [31], and higher than in the UK $(0.03 \%)$ [35] and Malaysia $(<0.001 \%)$ [33] (Table 4). Moreover, our findings showed that the mean level of EA in chocolate wafers were six times more than EA content in chocolate wafers in Malaysia [33]. As for the butter, the New Zealand [7] and Costa Rican butter [36] contained five times more EA, and the Pakistani butter [37] contained three more times EA, compared with our results (Table 4). However, the butter in UK, Germany, and Iran contained $0.22 \%$ [35], $0.23 \%$ [34], and $0.3 \%$ [27] EA respectively; this is lower than the content of EA tested in our study $(0.6 \%)$ (Table 4$)$. Also, Table 4 showed that the margarines in Slovenia contained the highest content of EA (34.63\%) [38] compared to our findings $(2.2 \%)$ and other countries. As for the EA content in chips, Iranian chips showed the highest level of EA (10\%) compared to our results $(0.1-0.3 \%)$ and other countries [27] (Table 4). On the other hand, the results of LEA in the food products tested in our study and those in other countries are available in Table 4.

\subsection{Comparison between Lebanese Market Basket Investigation and Other Global and Regional Market Investigations}

According to many studies, there was an impact of TFAs labeling on reducing the burden of CVDs due to TFAs [39]. According to an unpublished study conducted by our team, $32 \%$ only of the products available in the Lebanese markets reported TFAs on their labels (Data not shown). Our finding came to hand by hand with Kamel et al. [40], in which 181 food products were sampled from local supermarkets in Saudi Arabia and showed that one-third of the products mentioned TFAs on the nutrition label. Moreover, while the majority of the investigated samples in our project had low levels of TFAs, up to $14 \mathrm{~g}$ of TFAs per $100 \mathrm{~g}$ of food was observed in certain oils and fats sold at the Lebanese markets. Our findings, concerning the range of TFAs in-market products, were relatively low compared with the market investigations published in Stender et al. (2019-2020) [41,42].

\subsection{Investigation of the Country of Origin of Imported Food Products in Lebanon}

Lebanon imported its food products from France $(\$ 107,957)$, Germany $(\$ 98,250)$, Turkey $(\$ 97,015)$, United Kingdome $(\$ 75,571)$, Italy $(\$ 70,571)$, Argentina $(\$ 69,989)$, Saudi Arabia $(\$ 64$, 332 ) and United States $(\$ 57,785)$. In addition, the main importation sources of butter, oils, and fats are Denmark, Netherlands, France, Belgium, Ukraine, New Zealand, United Kingdom, 
and Argentina [43]. According to the nutrition labels of tested butter and margarines, the country of origin from which all the butter and margarines were imported to Lebanon were Turkey $(n=5)$, Egypt $(n=4)$, Malaysia $(n=3)$, Saudi Arabia $(n=1)$, Sri Lanka $(n=3)$, UAE $(n=1)$, Netherland $(n=2)$, Belgium $(n=3)$, France $(n=4)$, Italy $(n=1)$, Ukraine $(n=1)$, Germany $(n=2)$, and Denmark $(n=2)$. Among all these countries, 33 percent (five countries over 15) are implementing mandatory national limits and adopting monitoring mechanisms for mandatory of TFAs limits. On the other hand, in the remaining countries, the bestpractice TFAs policy passed but was not yet in effect [5]. Lebanon, long considered a middle-income country, is rapidly sinking into poverty as it faces a triple shock from the unprecedented economic crisis, the impact of COVID-19 on employment and public health, and the consequences of Beirut port explosions. Despite that, the actual relative impact of IP-TFAs exposure on heart disease mortality in Lebanon is limited, but unambiguously still considerable. The findings in our report highlight the importance of controlling the importation of food products from countries controlling IP-TFAs levels in food to avoid sinking Lebanese markets with IP-TFAs rich food products [44], both of which are often ultra-processed, unhealthy, and rich in IP-TFAs. Therefore, this population group is at higher risk of IP-TFAs-attributable CVDs.

\subsection{Limits, Advantages, and Future Directions}

This study presents some limitations. First, there are many challenges facing the laboratories in Lebanon concerning the testing of IP-TFA, and the lack of standards limits testing other forms of isomers. Second, the food products compared between regions were compared in terms of food groups and not in terms of brands. Moreover, the comparison between traditional dishes or Arabic sweets omits the cooking preparations and ingredients. Third, in the current study, the WHO technique was followed to test the IP-TFA levels in foods tested, however, this was not always reported in many other countries.

Despite these limitations, this study, the first of its kind in Lebanon, should provide the impetus for continuous comprehensive analysis of IP-TFA levels in foods in the regional and national kitchens and markets and the adaptation of the approaches for curbing the health hazards associated with IP-TFA consumption.

\section{Conclusions}

For the first time in Lebanon, a database on IP-TFA, mainly EA and LEA content in traditional dishes and market products is available and ready to be used by health care providers. There is more than enough convincing evidence that a high IP-TFAs, mainly EA and LEA intake is detrimental to cardiovascular health. Fortunately, this problem in Lebanon is fairly easy to solve via proper legislation. Despite the poorness of Lebanese dishes in IP-TFAs, however, the persistence of food products with high IP-TFAs levels in Lebanon means that subgroups of the Lebanese population, mainly vulnerable and foodinsecure people, are threatened by high levels of IP-TFAs due to frequent consumption of risky products. The inauguration and implementation of policies to curtail IP-TFAs in Lebanon may therefore be legitimized, and such efforts should underline added fats and packaged foods. The economic crises in Lebanon pushed the Lebanese people to select cheap oils, including butter and margarines instead of vegetable oils. Thus, it appears reasonable that the Lebanese government and ministries should strive to raise public awareness about the issue and lobby for implementing anti-IP-TFAs laws either on the level of national industries or, on the level of food products importation.

Author Contributions: Conceptualization, M.H. and E.Z.; methodology, M.H. and E.Z.; validation, A.A.-J. and I.S.; formal analysis, M.H. and C.I.; investigation, M.H., E.Z., C.I.; resources, E.Z., A.A.-J.; data curation, M.H., E.Z., C.I.; writing—original draft preparation, M.H., E.Z., C.I.; writing-review and editing, All the others; supervision, A.A.-J., A.R. and I.S. All authors have read and agreed to the published version of the manuscript.

Funding: This project was self-funded. 
Institutional Review Board Statement: Not applicable.

Informed Consent Statement: Not applicable.

Data Availability Statement: All data underlying the results are available as part of the article and no additional source data are required.

Acknowledgments: The authors would like to thank the head of the Chemical department Joseph Matta and the laboratory technician Halim El Bayeh at the Industrial Research Institute, the Research assistants. Nadia Hallak and Iman Kheir and the students Jessica Khalifeh and Layla Abadi at the Lebanese University. The authors confirm the approval of the acknowledged persons to be acknowledged.

Conflicts of Interest: The authors declare no conflict of interest.

Disclaimer: The authors alone are responsible for the views expressed in this article and they do not necessarily represent the views, decisions or policies of WHO or the other institutions with which the authors are affiliated.

\section{References}

1. World Health Organization-Noncommunicable Diseases (NCD) Country Profiles 2018. Available online: https://www.who.int/ publications/i/item/ncd-country-profiles-2018 (accessed on 2 June 2021).

2. Remig, V.; Franklin, B.; Margolis, S.; Kostas, G.; Nece, T.; Street, J.C. Trans fats in America: A review of their use, consumption, health implications, and regulation. J. Am. Diet. Assoc. 2010, 110, 585-592. [CrossRef] [PubMed]

3. World Health Organization. Countdown to 2023: WHO Report on Global Trans-Fat Elimination 2019; World Health Organization: Geneva, Switzerland, 2019.

4. World Health Organization. An Action Package to Eliminate Industrially-Produced Trans Fat from the Global Food Supply; World Health Organization: Geneva, Switzerland, 2018; Available online: http:/ /www.who.int/nutrition/topics/replace-transfat (accessed on 15 March 2021).

5. World Health Organization. Countdown to 2023: WHO Report on Global Trans-Fat Elimination 2020; World Health Organization: Geneva, Switzerland, 2020.

6. World Health Organization. REPLACE Trans Fat: An Action Package to Eliminate Industrially Produced Trans-Fatty Acids. In Module 3: Legislate or Regulate. How-to Guide for Trans Fat Policy Action; World Health Organization: Geneva, Switzerland, 2020; Licence: CC BY-NC-SA 3.0 IGO.

7. Saunders, D.; Jones, S.; Devane, G.J.; Scholes, P.; Lake, R.J.; Paulin, S.M. Trans fatty acids in the New Zealand food supply. J. Food Comp. Anal. 2008, 21, 320-325. [CrossRef]

8. Jawaldeh, A.A.; Al-Jawaldeh, H. Fat Intake Reduction Strategies among Children and Adults to Eliminate Obesity and NonCommunicable Diseases in the Eastern Mediterranean Region. Children 2018, 5, 89. [CrossRef] [PubMed]

9. World Health Organization. Noncommunicable Diseases: Key Facts. Geneva. 2018. Available online: https:/ /www.who.int/ news-room/fact-sheets/detail/noncommunicable-diseases (accessed on 16 March 2021).

10. Persistence Market Research. PHO and non-PHO Based Oils and Fats Market: Global Industry Analysis 2013-2017 and Forecast 2018-2026. Available online: https:/ / www.persistencemarketresearch.com/market-research/pho-and-nonpho-based-oils-andfats-market.asp (accessed on 17 June 2021).

11. Bösch, S.; Westerman, L.; Renshaw, N.; Pravst, I. Trans Fat Free by 2023-A Building Block of the COVID-19 Response. Front. Nutr. 2021, 8, 645750. [CrossRef]

12. Ohmori, H.; Fujii, K.; Kadochi, Y.; Mori, S.; Nishiguchi, Y.; Fujiwara, R.; Kishi, S.; Sasaki, T.; Kuniyasu, H. Elaidic Acid, a Trans-Fatty Acid, Enhances the Metastasis of Colorectal Cancer Cells. Pathobiol. J. Immunopathol. Mol. Cell. Biol. 2017, 84, 144-151. [CrossRef] [PubMed]

13. Li, C.; Cobb, L.K.; Vesper, H.W.; Asma, S. Global Surveillance of trans-Fatty Acids. Prev. Chronic Dis. 2019, 16, E147. [CrossRef]

14. Park, K.-H.; Kim, J.-M.; Cho, K.-H. Elaidic acid (EA) generates dysfunctional high-density lipoproteins and consumption of EA exacerbates hyperlipidemia and fatty liver change in zebrafish. Mol. Nutr. Food Res. 2014, 58, 1537-1545. [CrossRef]

15. Wang, J.; Liang, Y.; Jian, L.; Zhang, J.; Liang, S.; Xiao, S.; Liu, B.; Wang, H. Linoelaidic acid enhances adipogenic differentiation in adipose tissue-derived stromal cells through suppression of Wnt/ $\beta$-catenin signaling pathway in vitro. Prostaglandins Leukot. Essent. Fat. Acids 2016, 110, 1-7. [CrossRef]

16. Li, J.; Rao, H.; Bin, Q.; Fan, Y.-W.; Li, H.-Y.; Deng, Z.-Y. Linolelaidic acid induces apoptosis, cell cycle arrest and inflammation stronger than elaidic acid in human umbilical vein endothelial cells through lipid rafts. Eur. J. Lipid Sci. Technol. 2016, 119, 1600374. [CrossRef]

17. World Health Organization. Regional framework for action. In WHO/Noncommunicable Diseases; WHO Regional Office for Eastern Mediterranean: Cairo, Egypt, 2015.

18. Hoteit, M.; Zoghbi, E.; Al Iskandarani, M.; Rady, A.; Shankiti, I.; Matta, J.; Al-Jawaldeh, A. Nutritional value of the Middle Eastern diet: Analysis of total sugar, salt, and iron in Lebanese traditional dishes. F1000Research 2020, 9, 1254. [CrossRef] 
19. Hoteit, M.; Zoghbi, E.; Rady, A.; Shankiti, I.; Al-Jawaldeh, A. Development of a Lebanese food exchange system based on frequently consumed Eastern Mediterranean traditional dishes and Arabic sweets. F1000Research 2021, 10, 12. [CrossRef]

20. Food Composition Data: Traditional Dishes, Arabic Sweets and Market Foods. 2021. Available online: https://ul.edu.lb/files/ ann/20210422-LU-RePa-Report.pdf (accessed on 29 April 2021).

21. Batal, M.; Hunter, E. Traditional Lebanese recipes based on wild plants: An answer to diet simplification? Food Nutr. Bull. 2007, 28 (Suppl. 2), S303-S311. [CrossRef]

22. Issa, C.; Salameh, P.; Batal, M.; Vieux, F.; Lairon, D.; Darmon, N. The nutrient profile of traditional Lebanese composite dishes: Comparison with composite dishes consumed in France. Int. J. Food Sci. Nutr. 2009, 60 (Suppl. 4), 285-295. [CrossRef]

23. Association of Official Analytical Chemists. Official Methods of Analysis; Washington Ce 1h-05; 23; Association of Official Analytical Chemists: Washington, DC, USA, 2017; (Revised 2017).

24. Association of Official Analytical Chemists. Official Methods of Analysis, 21st ed.; Association of Official Analytical Chemists: Washington, DC, USA, 2019.

25. Bahrami, G.; Bahrami, S.; Bahrami, M.; Pasdar, Y.; Darbandi, M.; Rezaei, M. The Trans Fatty Acid Content of Snacks Offered in Kermanshah. Int. J. Health Life Sci. 2015, 1, e69803.

26. Wolff, R.L.; Combe, N.A.; Destaillats, F.; Boué, C.; Precht, D.; Molkentin, J.; Entressangles, B. Follow-up of the $\Delta 4$ to $\Delta 16$ trans-18:1 isomer profile and content in French processed foods containing partially hydrogenated vegetable oils during the period 1995-1999. Analytical and nutritional implications. Lipids 2000, 35, 815-825. [CrossRef]

27. Nazari, B.; Asgary, S.; Azadbakht, L. Fatty acid analysis of Iranian junk food, dairy, and bakery products: Special attention to trans-fats. J. Res. Med. Sci. Off. J. Isfahan Univ. Med. Sci. 2012, 17, 952-957.

28. Żbikowska, A.; Onacik-Gür, S.; Kowalska, M.; Rutkowska, J. Trans fatty acids in polish pastry. J. Food Prot. 2019, 82, 1028-1033.

29. Reshma, M.V.; Kiran, R.C.; Nisha, P.; SobanKumar, D.R.; Sundaresan, A.; Jayamurthy, P. Trans fat content in labeled and unlabelled indian bakery products including fried snacks. Int. Food Res. J. 2012, 19, 1609-1614. Available online: http:/ / ezproxy.usek.edu.lb/login?url=https:/ / www-proquest-com.ezproxy.usek.edu.lb/scholarly-journals / trans-fatcontent-labeled-unlabelled-indian/docview /1318005982/se-2?accountid=43544 (accessed on 2 July 2021).

30. Saadeh, C.; Toufeili, I.; Habbal, M.Z.; Nasreddine, L. Fatty acid composition including trans-fatty acids in selected cereal-based baked snacks from Lebanon. J. Food Compos. Anal. 2015, 41, 81-85. [CrossRef]

31. Lee, J.H.; Adhikari, P.; Kim, S.-A.; Yoon, T.; Kim, I.-H.; Lee, K.-T. Trans Fatty Acids Content and Fatty Acid Profiles in the Selected Food Products from Korea between 2005 and 2008. J. Food Sci. 2010, 75, C647-C652. [CrossRef]

32. Cakmak, Y.S.; Guler, G.O.; Yigit, S.; Caglav, G.; Aktumsek, A. Fatty Acid Composition and TransFatty Acids in Crisps and Cakes in Turkey's Markets. Int. J. Food Prop. 2011, 14, 822-829. [CrossRef]

33. Akmar, Z.D.; Norhaizan, M.E.; Azimah, R.; Azrina, A.; Chan, Y.M. The trans fatty acids content of selected foods in Malaysia. Malays. J. Nutr. 2013, 19, 87-98.

34. Kuhnt, K.; Baehr, M.; Rohrer, C.; Jahreis, G. Trans fatty acid isomers and the trans-9/trans11 index in fat containing foods. Eur. J. Lipid Sci. Technol. 2011, 113, 1281-1292. [CrossRef] [PubMed]

35. Roe, M.; Pinchen, H.; Church, S.; Elahi, S.; Walker, M.; Farron-Wilson, M.; Buttriss, J.; Finglas, P. Trans fatty acids in a range of UK processed foods. Food Chem. 2013, 140, 427-431. [CrossRef]

36. Baylin, A.; Siles, X.; Donovan-Palmer, A.; Fernandez, X.; Campos, H. Fatty acid composition of Costa Rican foods including trans fatty acid content. J. Food Compos. Anal. 2007, 20, 182-192. [CrossRef]

37. Anwar, F.; Bhanger, M.I.; Iqbal, S.; Sultana, B. Fatty acid composition of different margarines and butters from pakistan with special emphasis on trans unsaturated contents. J. Food Qual. 2006, 29, 87-96. [CrossRef]

38. Abramovič, H.; Vidrih, R.; Zlatić, E.; Kokalj, D.; Schreiner, M.; Žmitek, K.; Kušar, A.; Pravst, I. Trans fatty acids in margarines and shortenings in the food supply in Slovenia. J. Food Compos. Anal. 2018, 74, 53-61. [CrossRef]

39. Satia, J.A.; Galanko, J.A.; Neuhouser, M.L. Food nutrition label use is associated with demographic, behavioral, and psychosocial factors and dietary intake among African Americans in North Carolina. J. Am. Diet. Assoc. 2005, 105, 392-403. [CrossRef] [PubMed]

40. Kamel, S.; Otaibi, H.A. Trans-Fats Declaration, Awareness and Consumption in Saudi Arabia. Curr. Res. Nutr. Food Sci. 2018, 6, 748-756. [CrossRef]

41. Stender, S. Industrially produced trans fat in popular foods in 15 countries of the former Soviet Union from 2015 to 2016: A market basket investigation. BMJ Open 2019, 9, e023184. [CrossRef]

42. Stender, S. Trans fat in foods in Iran, South-Eastern Europe, Caucasia and Central Asia: A market basket investigation. Food Policy 2020, in press. [CrossRef]

43. Lebanon Dairy Produce; Butter and Other Fats and Oils Derived from Milk Imports by Country/2018/Data (worldbank.org). Available online: https://wits.worldbank.org/trade/comtrade/en/country/LBN/year/2018/tradeflow/Exports/partner/ ALL/product/040500 (accessed on 1 July 2021).

44. World Food Program. WFP Lebanon Country Brief February 2021. Available online: www.wfp.org/countries/Lebanon (accessed on 2 July 2021). 